\title{
Episodic Pain Syndrome, Familial, 3
}

National Cancer Institute

\section{Source}

National Cancer Institute. Episodic Pain Syndrome, Familial, 3. NCI Thesaurus. Code C125390.

A rare, autosomal dominant disorder caused by mutation in the SCN11A gene. It is characterized by intense episodic pain mainly affecting the distal lower extremities in early childhood. The pain diminishes with age. 